\title{
BOUNDARY-INTEGRAL CALCULATIONS OF TWO-DIMENSIONAL ELECTROMAGNETIC SCATTERING IN INFINITE PHOTONIC CRYSTAL SLABS: CHANNEL DEFECTS AND RESONANCES*
}

\author{
MANSOOR A. HAIDER ${ }^{\dagger}$, STEPHEN P. SHIPMAN $\ddagger$, AND STEPHANOS VENAKIDES ${ }^{\S}$
}

\begin{abstract}
We compute the transmission of two-dimensional (2D) electromagnetic waves through a square lattice of lossless dielectric rods with a channel defect. The lattice is finite in the direction of propagation of the incident wave and periodic in a transverse direction. We revisit a boundaryintegral formulation of 2D electromagnetic scattering [Venakides, Haider, and Papanicolaou, SIAM J. Appl. Math., 60 (2000), pp. 1686-1706] that is Fredholm of the first kind and develop a second-kind formulation. We refine the numerical implementation in the above paper by exploiting separability in the Green's function to evaluate the far-field influence more efficiently. The resulting cost savings in computing and solving the discretized linear system leads to an accelerated method. We use it to analyze $E$-polarized electromagnetic scattering of normally incident waves on a structure with a periodic channel defect. We find three categories of resonances: waveguide modes in the channel, high-amplitude fields in the crystal at frequencies near the edge of the frequency bandgap, and very high-amplitude standing fields at frequencies in a transmission band that are normal to the direction of the incident wave. These features are captured essentially identically with the first-kind as with the second-kind formulation.
\end{abstract}

Key words. EM scattering, photonic crystal, photonic bandgap, channel defect, boundary integral, boundary element method, Ewald representation, resonances

AMS subject classifications. 78, 45, 65, 42

PII. S003613990138531X

1. Introduction. Calculations of electromagnetic (EM) scattering in photonic crystal lattices play a central role in the design of optical and electronic devices including filters, lasers, and microwave antennas. The manner in which waves propagate through a photonic crystal is highly dependent on the geometry of the lattice, the loss properties of the component dielectrics, and the dielectric contrast. Under conditions of high dielectric contrast, it is well known that infinite two-dimensional (2D) lattices exhibit photonic bandgaps, which are frequency intervals in which there are no waves propagating through the lattice (see, e.g., [5], [9]).

In the practical case of a truncated photonic crystal, in particular a photonic crystal slab, the bandgaps appear as frequency intervals of very low transmission. When defects are introduced into the geometry, large fields at resonant frequencies arise in the structure, causing irregular behavior in the transmission coefficient at these frequencies.

In the present study, we consider EM scattering by a photonic crystal slab comprised of a square lattice of lossless dielectric rods. The slab is finite in one direction $X$ and periodic in the other direction $Y$. We first compute the effects of nonnormal

* Received by the editors February 21, 2001; accepted for publication (in revised form) December 19, 2001; published electronically July 24, 2002.

http://www.siam.org/journals/siap/62-6/38531.html

${ }^{\dagger}$ Department of Mathematics, North Carolina State University, Box 8205, Raleigh, NC 27695-8205 (mahaider@math.ncsu.edu). The research of this author was supported by NSF grant DMS-9705931.

${ }^{\ddagger}$ Department of Mathematics, Box 90320, Duke University, Durham, NC 27708-0320 (shipman@ math.duke.edu).

$\S$ Department of Mathematics, Box 90320, Duke University, Durham, NC 27708-0320 (ven@math. duke.edu). The research of this author was supported by grants ARO-DAAD19-99-1-0132 and NSF DMS-9500623. 
incidence and consider briefly the effect of random perturbations of the perfect lattice on the transmission coefficient. Then, as our primary focus, we analyze the scattering of normally incident waves by a structure with a periodic channel defect (Figure 4.3) that is parallel to the $X$-axis. We find three categories of resonances in the channel structure (Figure 4.5): waveguide modes in the channel at higher frequencies in the bandgap, high-amplitude 2D modes in the structure at lower frequencies in the bandgap, and very high-amplitude $Y$-resonating modes at frequencies to the left of the bandgap. The last category of resonances is of particular interest as such resonances have very high quality factors and exhibit characteristics similar to channel drop filters [2], [3], which reflect incident radiation at a particular frequency while allowing neighboring frequencies to penetrate the lattice.

In previous work [1], [8], we studied transmission through Fabry-Perot laser cavities formed by two parallel mirrors comprised of circular dielectric rods in air. In contrast to the channel in the present study, the Fabry-Perot cavity runs parallel to the $Y$ direction. In that case, resonant frequencies arise in the bandgap only. In [8], the location of peaks in the transmission graph and their quality factor ( $Q$-value), which measures laser efficiency, were traced as a function of cavity width for the case of lossless dielectrics. In the present study, we are concerned not only with characteristics in the transmission coefficient at various types of resonant frequencies in the band and the gap but also with the behavior of the electromagnetic field inside the structure at these frequencies.

For our studies, we use a numerical implementation of a boundary-integral formulation of the scattering problem that we developed in [8]. We also use a well-posed formulation that we develop in section 2.2. The boundary-integral approach reduces the problem from $2 \mathrm{D}$ to $1 \mathrm{D}$ and automatically enforces radiation conditions that are inherent to the scattering problem through the choice of appropriate radiating Green's functions. In this study, we refine our previous model by exploiting separability in the Green's function to evaluate the far-field influence more efficiently. As in the previous study, an Ewald representation of the Green's function is used to ensure rapid convergence of the representation in the near field. The primary consequence of our refined approach is that the (previously) dense matrix in the assembled system of linear equations is reduced to a banded matrix, resulting in an accelerated method. This simplification leads to significant cost savings in both computing and solving the discretized integral system.

2. The scattering problem and its mathematical formulation. We describe in this section our extension of the boundary-integral formulation of the electromagnetic scattering problem, which was developed in [8]. The photonic crystal under consideration is a mixed dielectric structure that is periodic in one direction $Y$ and finite in the other direction $X$. It is constant in the $Z$-direction. One period consists of a finite number of parallel rods $D_{j}$ transverse to the $X Y$-plane, each having a uniform dielectric permittivity $\epsilon_{j}$. We call the period of the structure a cell, or a supercell in the case in which it involves a finer periodic structure (Figure 2.1). The space exterior to the rods has a uniform dielectric permittivity $\epsilon_{\text {ext }}$. We refer to this structure as a photonic crystal slab.

We consider scattering by the structure of time-harmonic electric and magnetic fields with either electric or magnetic polarization. In the former, the electric field points parallel to the rods, and the magnetic field lies parallel to the $X Y$-plane, transverse to the rods. We refer to this as $E_{z}$-polarization. (In [8], these fields were called transverse magnetic, or TM, fields.) We define $H_{z}$-polarization (TE) in 


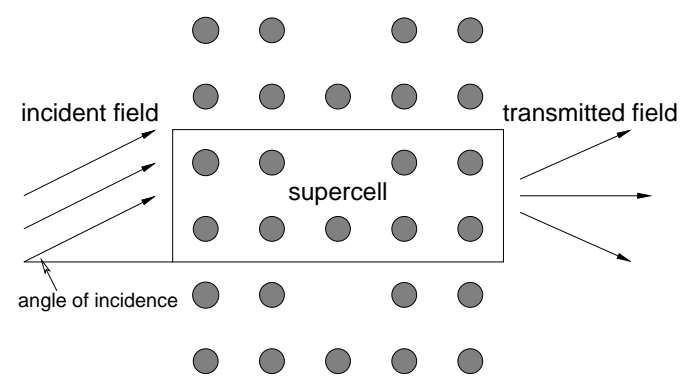

Fig. 2.1. A plane cross-section of a y-periodic photonic crystal. The supercell enclosed in a rectangle represents one period.

a corresponding fashion, with the magnetic field parallel to the rods. In either case, the field component parallel to the rods has the form $\Psi(X, Y) e^{-i \omega t}$, and $\Psi$ satisfies the Helmholtz equation

$$
\left(\partial_{X}^{2}+\partial_{Y}^{2}\right) \Psi+\frac{\omega^{2}}{c^{2}} \epsilon(X, Y) \Psi=0,
$$

in which $c$ is the speed of light. This equation is valid in the interiors of the rods and in the exterior, and matching conditions described below apply on the interface. Because of the periodicity of the structure, the domain of the mathematical problem is the strip $\{(X, Y):-\infty<X<\infty, 0 \leq Y \leq P\}$, where $P$ is the period.

2.1. Equations for the electromagnetic field. In dimensionless form, we scale the period to $2 \pi$, and we let $\psi(x, y)$ denote the electric $\left(E_{z}\right.$ case) or magnetic field $\left(H_{z}\right.$ case). Here $\psi$ satisfies the nondimensional Helmholtz equation

$$
\left(\partial_{x}^{2}+\partial_{y}^{2}\right) \psi+k^{2} \epsilon(x, y) \psi=0,
$$

in which $k$, the reduced (nondimensional) frequency, is given by $k=\omega p /(2 \pi c)$, where $p$ is the $Y$-period of the structure. $\epsilon=\epsilon_{\text {int }} / \epsilon_{\text {ext }}$ is the dielectric contrast between the interior and the exterior and is equal to 1 in the exterior to the rods. The following conditions also apply:

- The field $\psi_{\text {ext }}$ in the exterior is the sum of an incident plane wave $\psi_{\text {inc }}$ and a scattered wave $\psi_{\mathrm{sc}}$.

- $\psi_{\mathrm{sc}}$ satisfies a radiation condition at $x= \pm \infty$.

- Let $\psi_{\text {int }}$ denote the field inside $\operatorname{rod} D_{j}$. On the boundary $\partial D_{j}$, the following matching conditions hold:

$$
\psi_{\mathrm{ext}}=\psi_{\mathrm{inc}}+\psi_{\mathrm{sc}}=\psi_{\mathrm{int}}, \quad \partial_{n} \psi_{\mathrm{ext}}=\partial_{n} \psi_{\mathrm{inc}}+\partial_{n} \psi_{\mathrm{sc}}=\nu^{-1} \partial_{n} \psi_{\mathrm{int}},
$$

where $\nu=1$ for $E_{z}$ waves and $\nu=\epsilon$ for $H_{z}$ waves.

If the incident field is produced at an angle of $\theta$ with the normal to the surface of the crystal ( $x$-axis), then, by requiring that it satisfy the Helmholtz equation (2.1), we find that it has the form

$$
\psi_{\mathrm{inc}}=\exp \left(i \sqrt{k^{2}-(\bar{m}+\beta)^{2}} x+i(\bar{m}+\beta) y\right),
$$

in which $\theta=\arcsin ((\bar{m}+\beta) / k), \bar{m}$ is an integer, and $-1 / 2<\beta \leq 1 / 2$. $\psi_{\text {inc }}$ is pseudoperiodic in the sense that

$$
\psi_{\mathrm{inc}}=e^{i \beta y} \tilde{\psi}_{\mathrm{inc}}(x, y),
$$


where $\tilde{\psi}_{\text {inc }}$ is $2 \pi$-periodic in $\_$. Requiring that the scattered field also be pseudoperiodic, so that $\psi_{\mathrm{sc}}=e^{i \beta y} \tilde{\psi}_{\mathrm{sc}}(x, y)$, where $\tilde{\psi}_{\mathrm{sc}}$ is $2 \pi$-periodic in $y$, we find that, in the exterior,

$$
\left(\partial_{x}^{2}+\partial_{y}^{2}\right) \tilde{\psi}_{\mathrm{sc}}+2 i \beta \partial_{y} \tilde{\psi}_{\mathrm{sc}}+\left(k^{2}-\beta^{2}\right) \tilde{\psi}_{\mathrm{sc}}=0
$$

The radiating Green's function for the exterior is then the pseudoperiodic function

$$
G(\mathbf{r}-\hat{\mathbf{r}})=e^{i \beta(y-\hat{y})} \tilde{G}(\mathbf{r}-\hat{\mathbf{r}}),
$$

in which $\tilde{G}$ is the field at the point $\mathbf{r}=(x, y)$ produced by a radiating periodic monopole of (2.3):

$$
\tilde{G}\left(\mathbf{r}-\hat{\mathbf{r}} ; k^{2}, \beta\right)=\frac{1}{4 \pi} \sum_{m=-\infty}^{\infty} \frac{e^{-\sqrt{-\lambda_{m}}|x-\hat{x}|}}{\sqrt{-\lambda_{m}}} e^{i m(y-\hat{y})},
$$

where $\lambda_{m}=k^{2}-(m+\beta)^{2}$.

Considering the matching conditions (2.2), the radiation condition on $\psi_{\mathrm{sc}}$, and the pseudoperiodicity of the incident and scattered fields and the Green's function, Green's identity results in the following system of boundary-integral equations for the total exterior field $\psi_{\text {ext }}$ and its normal derivative $\partial_{n} \psi_{\text {ext }}:{ }^{1}$ For any point $\hat{\mathbf{r}} \in \partial D_{j}$,

$$
\begin{gathered}
\frac{1}{2} \psi_{\text {ext }}(\hat{\mathbf{r}})+\int_{\partial D}\left[-\frac{\partial G(\hat{\mathbf{r}}-\mathbf{r})}{\partial n(\mathbf{r})} \psi_{\mathrm{ext}}(\mathbf{r})+G(\hat{\mathbf{r}}-\mathbf{r}) \frac{\partial \psi_{\mathrm{ext}}}{\partial n(\mathbf{r})}(\mathbf{r})\right] d s(\mathbf{r})=\psi_{\mathrm{inc}}(\hat{\mathbf{r}}), \\
\frac{1}{2} \psi_{\mathrm{ext}}(\hat{\mathbf{r}})+\int_{\partial D_{j}}\left[\frac{\partial \Phi_{j}(\hat{\mathbf{r}}-\mathbf{r})}{\partial n(\mathbf{r})} \psi_{\mathrm{ext}}(\mathbf{r})-\nu \Phi_{j}(\hat{\mathbf{r}}-\mathbf{r}) \frac{\partial \psi_{\mathrm{ext}}}{\partial n(\mathbf{r})}(\mathbf{r})\right] d s(\mathbf{r})=0
\end{gathered}
$$

in which $\Phi_{j}$ is a Green's function for the interior of $\operatorname{rod} j$ and $\partial D$ is the union of all $\partial D_{j}$.

This system (2.5) is Fredholm of the first kind in the functional variable $\partial_{n} \psi_{\text {ext }}$.

2.2. A well-posed formulation of the boundary-integral equations. We have developed a well-posed formulation of the problem, in which the boundaryintegral equations are Fredholm of the second kind.

THEOREM 2.1. Suppose that the matching conditions (2.2), the radiating condition on $\psi_{\mathrm{sc}}$, and the pseudoperiodicity of $\psi_{\mathrm{sc}}$ and $\psi_{\mathrm{inc}}$ are satisfied. Then the following system of integral equations for $\psi_{\text {ext }}$ holds:

$$
\begin{aligned}
\psi_{\text {ext }}(\hat{\mathbf{r}})+\int_{\partial D}\left[\frac{\partial(\Phi-G)(\hat{\mathbf{r}}-\mathbf{r})}{\partial n(\mathbf{r})} \psi_{\text {ext }}(\mathbf{r})-(\nu \Phi-G)(\hat{\mathbf{r}}-\mathbf{r}) \frac{\partial \psi_{\text {ext }}}{\partial n(\mathbf{r})}(\mathbf{r})\right] d s(\mathbf{r})=\psi_{\text {inc }}(\hat{\mathbf{r}}), \\
\begin{aligned}
\frac{1+\nu}{2} \frac{\partial \psi_{\text {ext }}}{\partial n(\hat{\mathbf{r}})}(\hat{\mathbf{r}})+\int_{\partial D}[ & \frac{\partial^{2}(\Phi-G)(\hat{\mathbf{r}}-\mathbf{r})}{\partial n(\hat{\mathbf{r}}) \partial n(\mathbf{r})} \psi_{\text {ext }}(\mathbf{r}) \\
& \left.-\frac{\partial(\nu \Phi-G)(\hat{\mathbf{r}}-\mathbf{r})}{\partial n(\hat{\mathbf{r}})} \frac{\partial \psi_{\text {ext }}}{\partial n(\mathbf{r})}(\mathbf{r})\right] d s(\mathbf{r})=\frac{\partial \psi_{\text {inc }}}{\partial n(\hat{\mathbf{r}})}(\hat{\mathbf{r}}) .
\end{aligned}
\end{aligned}
$$

\footnotetext{
${ }^{1}$ In [8], an extra term with a factor of $\beta$ appears erroneously in equation 4.6 and propagates into equations $4.12,4.15$, and 5.6. The error does not affect the results of that paper because only the case $\beta=0$ is considered. The authors apologize for the error.
} 
The notation $\partial / \partial n(\mathbf{r})$ refers to differentiation with respect to the variable $\mathbf{r}$ in the normal direction to the boundary at the point $\mathbf{r}$, and $\partial / \partial n(\hat{\mathbf{r}})$ refers to differentiation with respect to the variable $\hat{\mathbf{r}}$ in the normal direction to the boundary at the point $\hat{\mathbf{r}}$. It is also understood that the interior Green's function $\Phi(\hat{\mathbf{r}}-\mathbf{r})$ is zero whenever $\mathbf{r}$ and $\hat{\mathbf{r}}$ are on different rods.

These equations can be generalized to a system in which the scatterer has a magnetic permeability that is different from the external medium.

Similar formulations for 3D EM scattering problems are derived in Müller [7]. Our formulation is derived below from the first-kind system (2.5).

Proof. We start with the equation for the external field for values of $\hat{\mathbf{r}}$ in the domain exterior to the rods:

$$
\psi_{\text {ext }}(\hat{\mathbf{r}})+\int_{\partial D}\left[-\frac{\partial G(\hat{\mathbf{r}}-\mathbf{r})}{\partial n(\mathbf{r})} \psi_{\mathrm{ext}}(\mathbf{r})+G(\hat{\mathbf{r}}-\mathbf{r}) \frac{\partial \psi_{\mathrm{ext}}}{\partial n(\mathbf{r})}(\mathbf{r})\right] d s(\mathbf{r})=\psi_{\mathrm{inc}}(\hat{\mathbf{r}}) .
$$

Let $\hat{\mathbf{r}}$ lie on a vector $n(\hat{\mathbf{r}})$ emanating normally from some point on the boundary of one of the rods, and take the derivative of this equation with respect to $\hat{\mathbf{r}}$ in the direction of $n(\hat{\mathbf{r}})$ :

$$
\frac{\partial \psi_{\text {ext }}}{\partial n(\hat{\mathbf{r}})}(\hat{\mathbf{r}})+\int_{\partial D}\left[-\frac{\partial^{2} G(\hat{\mathbf{r}}-\mathbf{r})}{\partial n(\hat{\mathbf{r}}) \partial n(\mathbf{r})} \psi_{\text {ext }}(\mathbf{r})+\frac{\partial G(\hat{\mathbf{r}}-\mathbf{r})}{\partial n(\hat{\mathbf{r}})} \frac{\partial \psi_{\text {ext }}}{\partial n(\mathbf{r})}(\mathbf{r})\right] d s(\mathbf{r})=\frac{\partial \psi_{\text {inc }}}{\partial n(\hat{\mathbf{r}})}(\hat{\mathbf{r}}) .
$$

The following identity holds:

$$
\frac{\partial^{2} G(\hat{\mathbf{r}}-\mathbf{r})}{\partial n(\hat{\mathbf{r}}) \partial n(\mathbf{r})}+\frac{\partial^{2} G(\hat{\mathbf{r}}-\mathbf{r})}{\partial t(\hat{\mathbf{r}}) \partial t(\mathbf{r})}=-n(\hat{\mathbf{r}}) \cdot n(\mathbf{r})\left(\partial_{x}^{2}+\partial_{y}^{2}\right) G(\hat{\mathbf{r}}-\mathbf{r}),
$$

in which $\partial t$ denotes a tangent derivative. The minus sign occurs because $\partial n(\hat{\mathbf{r}})$ and $\partial t(\hat{\mathbf{r}})$ denote derivatives with respect to $\hat{\mathbf{r}}$. Using this together with the Helmholtz equation

$$
\left(\partial_{x}^{2}+\partial_{y}^{2}\right) G(\hat{\mathbf{r}}-\mathbf{r})+k^{2} G(\hat{\mathbf{r}}-\mathbf{r})=0 \quad \text { for } \hat{\mathbf{r}} \neq \mathbf{r},
$$

we eliminate the term containing two normal derivatives of $G$. Then, integrating by parts in the term with the tangent derivatives, we obtain

$$
\begin{aligned}
\frac{\partial \psi_{\text {ext }}}{\partial n(\hat{\mathbf{r}})}(\hat{\mathbf{r}})+\int_{\partial D}[ & -n(\hat{\mathbf{r}}) \cdot n(\mathbf{r}) k^{2} G(\hat{\mathbf{r}}-\mathbf{r}) \psi_{\text {ext }}(\mathbf{r}) \\
& \left.-\frac{\partial G(\hat{\mathbf{r}}-\mathbf{r})}{\partial t(\hat{\mathbf{r}})} \frac{\partial \psi_{\text {ext }}}{\partial t(\mathbf{r})}(\mathbf{r})+\frac{\partial G(\hat{\mathbf{r}}-\mathbf{r})}{\partial n(\hat{\mathbf{r}})} \frac{\partial \psi_{\text {ext }}}{\partial n(\mathbf{r})}(\mathbf{r})\right] d s(\mathbf{r})=\frac{\partial \psi_{\text {inc }}}{\partial n(\hat{\mathbf{r}})}(\hat{\mathbf{r}})
\end{aligned}
$$

We now allow $\hat{\mathbf{r}}$ to approach the boundary along the vector $n(\hat{\mathbf{r}})$, and the term con-

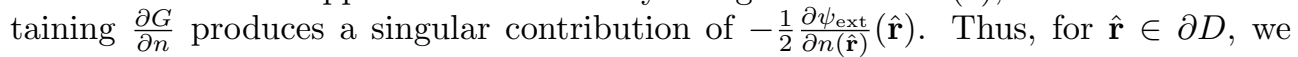
obtain

$$
\begin{aligned}
\frac{1}{2} \frac{\partial \psi_{\text {ext }}}{\partial n(\hat{\mathbf{r}})}(\hat{\mathbf{r}})+\int_{\partial D}[ & -n(\hat{\mathbf{r}}) \cdot n(\mathbf{r}) k^{2} G(\hat{\mathbf{r}}-\mathbf{r}) \psi_{\text {ext }}(\mathbf{r}) \\
& \left.-\frac{\partial G(\hat{\mathbf{r}}-\mathbf{r})}{\partial t(\hat{\mathbf{r}})} \frac{\partial \psi_{\text {ext }}}{\partial t(\mathbf{r})}(\mathbf{r})+\frac{\partial G(\hat{\mathbf{r}}-\mathbf{r})}{\partial n(\hat{\mathbf{r}})} \frac{\partial \psi_{\text {ext }}}{\partial n(\mathbf{r})}(\mathbf{r})\right] d s(\mathbf{r})=\frac{\partial \psi_{\mathrm{inc}}}{\partial n(\hat{\mathbf{r}})}(\hat{\mathbf{r}}) .
\end{aligned}
$$


A similar derivation applies to the field in the interior of the rods:

$$
\begin{aligned}
\frac{1}{2} \frac{\partial \psi_{\text {int }}}{\partial n(\hat{\mathbf{r}})}(\hat{\mathbf{r}})+\int_{\partial D}[ & n(\hat{\mathbf{r}}) \cdot n(\mathbf{r}) k^{2} \epsilon \Phi(\hat{\mathbf{r}}-\mathbf{r}) \psi_{\text {int }}(\mathbf{r}) \\
& \left.+\frac{\partial \Phi(\hat{\mathbf{r}}-\mathbf{r})}{\partial t(\hat{\mathbf{r}})} \frac{\partial \psi_{\text {int }}}{\partial t(\mathbf{r})}(\mathbf{r})-\frac{\partial \Phi(\hat{\mathbf{r}}-\mathbf{r})}{\partial n(\hat{\mathbf{r}})} \frac{\partial \psi_{\text {int }}}{\partial n(\mathbf{r})}(\mathbf{r})\right] d s(\mathbf{r})=0 .
\end{aligned}
$$

The integral of the term containing the tangent derivative is a principal-value integral. The leading order of this singularity, however, is independent of $k$ and $\epsilon$. Substituting $\psi_{\text {int }}=\psi_{\text {ext }}$ and $\partial_{n} \psi_{\text {int }}=\nu \partial_{n} \psi_{\text {ext }}$ from conditions (2.2) into (2.9) and adding (2.8), we obtain

$$
\begin{aligned}
& \frac{1+\nu}{2} \frac{\partial \psi_{\text {ext }}}{\partial n(\hat{\mathbf{r}})}(\hat{\mathbf{r}})+\int_{\partial D}\left[-n(\hat{\mathbf{r}}) \cdot n(\mathbf{r}) k^{2}(G-\epsilon \Phi)(\hat{\mathbf{r}}-\mathbf{r}) \psi_{\text {ext }}(\mathbf{r})\right. \\
& \left.\quad-\frac{\partial(G-\Phi)(\hat{\mathbf{r}}-\mathbf{r})}{\partial t(\hat{\mathbf{r}})} \frac{\partial \psi_{\text {ext }}}{\partial t(\mathbf{r})}(\mathbf{r})+\frac{\partial(G-\nu \Phi)(\hat{\mathbf{r}}-\mathbf{r})}{\partial n(\hat{\mathbf{r}})} \frac{\partial \psi_{\text {ext }}}{\partial n(\mathbf{r})}(\mathbf{r})\right] d s(\mathbf{r})=\frac{\partial \psi_{\text {inc }}}{\partial n(\hat{\mathbf{r}})}(\hat{\mathbf{r}}) .
\end{aligned}
$$

This equation contains no principal-value integral. In addition, the leading singular part of $G-\Phi$ is $C|\hat{\mathbf{r}}-\mathbf{r}|^{2} \log |\hat{\mathbf{r}}-\mathbf{r}|$, and we may therefore integrate again by parts. This yields the equation

$$
\begin{aligned}
& \frac{1+\nu}{2} \frac{\partial \psi_{\text {ext }}}{\partial n(\hat{\mathbf{r}})}(\hat{\mathbf{r}})+\int_{\partial D}\left[-n(\hat{\mathbf{r}}) \cdot n(\mathbf{r}) k^{2}(G-\epsilon \Phi)(\hat{\mathbf{r}}-\mathbf{r}) \psi_{\text {ext }}(\mathbf{r})\right. \\
& \left.\quad+\frac{\partial^{2}(G-\Phi)(\hat{\mathbf{r}}-\mathbf{r})}{\partial t(\hat{\mathbf{r}}) \partial t(\mathbf{r})} \psi_{\text {ext }}(\mathbf{r})+\frac{\partial(G-\nu \Phi)(\hat{\mathbf{r}}-\mathbf{r})}{\partial n(\hat{\mathbf{r}})} \frac{\partial \psi_{\text {ext }}}{\partial n(\mathbf{r})}(\mathbf{r})\right] d s(\mathbf{r})=\frac{\partial \psi_{\text {inc }}}{\partial n(\hat{\mathbf{r}})}(\hat{\mathbf{r}}) .
\end{aligned}
$$

The first two terms in the integral can now be replaced by

$$
-\frac{\partial^{2}(G-\Phi)(\hat{\mathbf{r}}-\mathbf{r})}{\partial n(\hat{\mathbf{r}}) \partial n(\mathbf{r})} \psi_{\text {ext }}(\mathbf{r})
$$

resulting in the second equation stated in the theorem.

The first equation is just the sum of (2.5).

2.3. The Ewald formulation of the Green's function. The Ewald formulation of the Green's function allows one to compute $G$ and its derivatives for small values of $|\hat{x}-x|$ for which the Fourier form (2.4) converges slowly. One writes $G$ as a sum

$$
G(\mathbf{r}-\hat{\mathbf{r}})=G_{1}(\mathbf{r}-\hat{\mathbf{r}})+G_{2}(\mathbf{r}-\hat{\mathbf{r}}),
$$

in which

$$
\begin{aligned}
& G_{1}=\frac{1}{2 \pi} \sum_{m=-\infty}^{\infty} e^{i(m+\beta)(y-\hat{y})} \int_{0}^{E^{2}} \frac{1}{\sqrt{4 \pi t}} \exp \left[\lambda_{m} t-\frac{(x-\hat{x})^{2}}{4 t}\right] d t \\
& G_{2}=\frac{1}{2 \pi} \sum_{m=-\infty}^{\infty} e^{i(m+\beta)(y-\hat{y})} \int_{E^{2}}^{\infty} \frac{1}{\sqrt{4 \pi t}} \exp \left[\lambda_{m} t-\frac{(x-\hat{x})^{2}}{4 t}\right] d t
\end{aligned}
$$


and the number $E$ is to be chosen judiciously. Following [8], we fix $E|k|=1$. In [8], $G_{1}$ is further reduced to the form

$$
G_{1}(\mathbf{r}-\hat{\mathbf{r}})=\frac{1}{4 \pi} \sum_{m=-\infty}^{\infty} e^{-2 \pi i \beta m} \sum_{n=0}^{\infty} \frac{(E k)^{2 n}}{n !} E_{n+1}\left(\frac{R_{m}^{2}}{4 E^{2}}\right)
$$

in which $R_{m}^{2}=(x-\hat{x})^{2}+(y-\hat{y}+2 \pi m)^{2}$ and $E_{n}(z)$ is the exponential integral of degree $n$ :

$$
E_{n}(z)=\int_{1}^{\infty} \frac{e^{-z t}}{t^{n}} d t
$$

By expanding $\exp \left((x-\hat{x})^{2} / 4 t\right)$ in a Taylor series, we reduce $G_{2}$ to the form

$$
G_{2}(\mathbf{r}-\hat{\mathbf{r}})=\frac{1}{2 \pi} \sum_{m=-\infty}^{\infty} e^{i(m+\beta)(\hat{y}-y)} \sum_{n=0}^{\infty} c_{m, n}(\hat{x}-x)^{2 n},
$$

in which

$$
c_{m, n}=\left(E^{2\left(n-\frac{1}{2}\right)} \sqrt{4 \pi}(-4)^{n} n !\right)^{-1} E_{n+\frac{1}{2}}\left(-E^{2} \lambda_{m}\right) .
$$

For any value of $k$, we calculate a sufficient number of these coefficients once and then use them to calculate the inner sum, for any values of $\hat{x}$ and $x$, to great precision. We find that this approach very significantly increases the efficiency in the calculations over the reduction of $G_{2}$ in [8] involving error functions.

The utility of the Ewald formulation arises from two features. First, the logarithmic singularity of $G$ is conveniently isolated in the term $m=0$ of $G_{1}$, and the leading-order part occurs in the term $n=0$ of that term. Separating the logarithmic and regular parts of the exponential integrals in this term, one can then perform integrations using logarithmic Gaussian quadrature rules when $|\hat{\mathbf{r}}-\mathbf{r}|$ is small. Second, the convergence of $G_{2}$ is exponential, uniformly in $|\hat{x}-x|$.

The exponential integrals are calculated in the following way: $E_{1}$ is calculated using polynomial and rational approximations, and $E_{1 / 2}$ using the identity

$$
E_{1 / 2}(z)=\sqrt{\frac{\pi}{z}} \operatorname{erfc}(\sqrt{z})
$$

and polynomial approximations for the complex complementary error function (erfc):

$$
\operatorname{erfc}(z)=\frac{2}{\sqrt{\pi}} \int_{z}^{\infty} e^{-t^{2}} d t
$$

Then, for $n>1, E_{n}(z)$ and $E_{n+1 / 2}(z)$ and their derivatives are calculated using the recursion relation

$$
E_{n+1}(z)=\frac{1}{n}\left(e^{-z}-z E_{n}(z)\right), \quad \frac{d E_{n}}{d z}(z)=-E_{n-1}(z) .
$$

In addition, near the logarithmic singularity in $G$, we split $E_{n}$ as follows:

$$
E_{n}(z)=E_{n}^{\mathrm{sing}}(z) \log (z)+E_{n}^{\mathrm{reg}}(z) .
$$

Again, $E_{1}^{\text {reg }}$ is calculated using polynomial and rational approximations, and $E_{1}^{\text {sing }}=$ -1 . Then, for $n>0, E_{n}^{\text {reg }}$ satisfies the same recursion relation as $E_{n}$, and $E_{n}^{\operatorname{sing}}(z)$ satisfies

$$
E_{n+1}^{\operatorname{sing}}(z)=-\frac{z}{n} E_{n}^{\operatorname{sing}}(z) .
$$


3. Numerical calculations. We have presented two formulations of the boundary-integral equations, one that is numerically ill-posed (2.5) and one that is well-posed (2.6). We have implemented both of these numerically to simulate a variety of experiments. We compare the results of the two schemes in section 4.2.

We now present our numerical scheme for the first-kind system. It can just as well be applied to the second-kind system.

3.1. The finite approximating system. Define, for $\hat{\mathbf{r}} \in \partial D_{j}$ and functions $\psi$ and $\phi$ defined on $\partial D$,

$$
\begin{aligned}
& L_{1}(\psi, \phi)(\hat{\mathbf{r}})=\frac{1}{2} \psi(\hat{\mathbf{r}})+\int_{\partial D}\left[-\frac{\partial G(\hat{\mathbf{r}}-\mathbf{r})}{\partial n(\mathbf{r})} \psi(\mathbf{r})+G(\hat{\mathbf{r}}-\mathbf{r}) \phi(\mathbf{r})\right] d s(\mathbf{r}), \\
& L_{2}(\psi, \phi)(\hat{\mathbf{r}})=\frac{1}{2} \psi(\hat{\mathbf{r}})+\int_{\partial D_{j}}\left[\frac{\partial \Phi_{j}(\hat{\mathbf{r}}-\mathbf{r})}{\partial n(\mathbf{r})} \psi(\mathbf{r})-\nu \Phi_{j}(\hat{\mathbf{r}}-\mathbf{r}) \phi(\mathbf{r})\right] d s(\mathbf{r}) .
\end{aligned}
$$

We choose a finite-dimensional approximation space for the space of continuous functions on $\partial D$ spanned by basis functions $\left\{h_{i}\right\}_{i=1}^{N}$ and also choose $N$ sample points $\left\{\hat{\mathbf{r}}_{j}\right\}_{j=1}^{N}$. Let the incident and external fields be approximated by

$$
\begin{aligned}
\left(\psi_{\mathrm{inc}}, \partial_{n} \psi_{\mathrm{inc}}\right) & \approx\left(\sum_{i=1}^{N} c_{i} h_{i}, \sum_{i=1}^{N} d_{i} h_{i}\right), \\
\left(\psi_{\mathrm{sc}}, \partial_{n} \psi_{\mathrm{sc}}\right) & \approx\left(\sum_{i=1}^{N} a_{i} h_{i}, \sum_{i=1}^{N} b_{i} h_{i}\right) .
\end{aligned}
$$

We then arrive at an approximating finite-dimensional linear system for the system (2.5):

$$
\begin{aligned}
& L_{1}\left(\sum a_{i} h_{i}, \sum b_{i} h_{i}\right)\left(\hat{\mathbf{r}}_{j}\right)=\psi_{\mathrm{inc}}\left(\hat{\mathbf{r}}_{j}\right), \quad j=1, \ldots, N, \\
& L_{2}\left(\sum a_{i} h_{i}, \sum b_{i} h_{i}\right)\left(\hat{\mathbf{r}}_{j}\right)=0, \quad j=1, \ldots, N .
\end{aligned}
$$

This is a $2 N \times 2 N$ linear system for $\left\{a_{i}, b_{i}\right\}_{i=1}^{N}$.

For all of the calculations in this paper, we have exactly parameterized the boundaries of the rods, divided each boundary into a number of elements, and then used basis functions $\left\{h_{i}\right\}$ that correspond to three-node quadratic interpolation of the fields along the elements. Thus there are two types of basis functions - one that is supported on a single element, and another that is supported on two adjacent elements. The numbers $\left\{a_{i}, b_{i}\right\}$ and $\left\{c_{i}, d_{i}\right\}$ are the values of the fields $\psi_{\text {ext }}$ and $\psi_{\text {inc }}$ at the endpoints and midpoints of each element. As sample points $\left\{\hat{r}_{j}\right\}$, we have again chosen to use the endpoints and midpoints of the elements. In computing the matrix elements of the system (3.1), we have used regular Gaussian quadrature formulas for the integrals over those elements for which the integrand has no singularity. For elements whose support contains the sample point $\hat{r}_{j}$, we use Gaussian quadrature formulas with logarithmic weights to compute the integrals of the singular term $m=0$ of $G_{1}$.

3.2. An accelerated computational scheme. Each term of the Fourier form of the periodic Green's function $G(\hat{\mathbf{r}}-\mathbf{r})$ and its derivatives can be written as a product of a factor involving only $\hat{x}$ and $\hat{y}$ with a factor involving only $x$ and $y$. This separability is accompanied by exponential decay in $m$ when $|x-\hat{x}|$ is not zero. When 


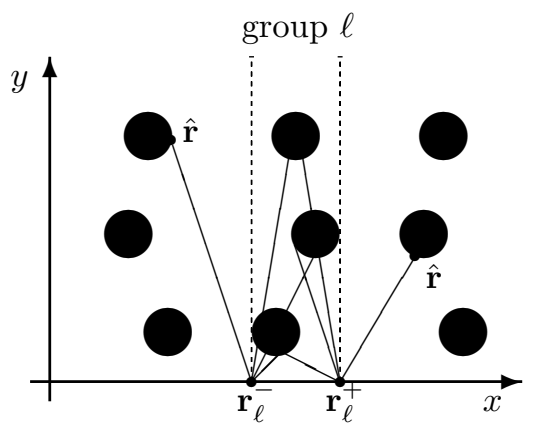

FIG. 3.1. The separability of the modes of the periodic Green's function: the influence of one group of rods on a remote point $\hat{\mathbf{r}}$ through the reference points $\mathbf{r}_{\ell}^{ \pm}$.

$|x-\hat{x}|$ is sufficiently large, this decay allows us to consider only a small number of terms of the series, while the separability allows us to group influences as described below. The computational advantage of this grouping is that it can be utilized to lead to a sparse matrix in the final linear algebraic system. When $|x-\hat{x}|$ is small, we use the Ewald representation. Although it displays fast convergence, as we discussed above, we lose the advantage of separability in this regime. Our method is thus much more efficient for structures with a supercell that is long in the $x$ direction and short in the $y$ direction, and least efficient in the opposite case.

We partition the set of rods into $L$ vertical groups and denote by $D_{\ell}$ the domain consisting of the rods in group $\ell$ (Figure 3.1). Set $x_{\ell}^{+}=\sup \left\{x:(x, y) \in \partial D_{\ell}\right\}$, $x_{\ell}^{-}=\inf \left\{x:(x, y) \in \partial D_{\ell}\right\}$, and $\mathbf{r}_{\ell}^{ \pm}=\left(x_{\ell}^{ \pm}, 0\right)$. For each $\ell=1, \ldots, L$ and each integer $m$, define

$$
\begin{aligned}
L_{m, \ell}^{+}(\psi, \phi)= & \frac{1}{4 \pi \sqrt{-\lambda_{m}}} \sum_{\ell^{\prime}=0}^{\ell} \int_{\partial D_{\ell^{\prime}}} e^{-\sqrt{-\lambda_{m}}\left|x_{\ell}^{+}-x\right|-i(m+\beta) y} \\
& \times\left[\phi(\mathbf{r})+\left(-\sqrt{-\lambda_{m}} n_{x}(\mathbf{r})+i(m+\beta) n_{y}(\mathbf{r})\right) \psi(\mathbf{r})\right] d s(\mathbf{r}), \\
L_{m, \ell}^{-}(\psi, \phi)= & \frac{1}{4 \pi \sqrt{-\lambda_{m}}} \sum_{\ell^{\prime}=\ell}^{L} \int_{\partial D_{\ell^{\prime}}} e^{-\sqrt{-\lambda_{m}}\left|x_{\ell}^{-}-x\right|-i(m+\beta) y} \\
& \times\left[\phi(\mathbf{r})+\left(\sqrt{-\lambda_{m}} n_{x}(\mathbf{r})+i(m+\beta) n_{y}(\mathbf{r})\right) \psi(\mathbf{r})\right] d s(\mathbf{r}),
\end{aligned}
$$

We call $\mathbf{r}_{\ell}^{ \pm}$the right and left reference points of influence for group $\ell ; \mathbf{r}$ is a source point, and $\hat{\mathbf{r}}$ is the influence point. $L_{m, \ell}^{ \pm}\left(\psi, \partial_{n} \psi\right)$ represents the contribution of source points located on $\partial D_{\ell}$ to the $m$ th Fourier mode of the field at the reference point $\mathbf{r}_{\ell}^{ \pm}$. See Figure 3.1 for an illustration.

Then, given $\hat{\mathbf{r}} \in \partial D$ such that $x<\hat{x}$ for all $(x, y) \in \partial D_{\ell}$, the integral in (2.5a), restricted to $\bigcup_{\ell^{\prime}<\ell} \partial D_{\ell}$, becomes

$$
\begin{gathered}
\sum_{\ell^{\prime}=1}^{\ell} \int_{\partial D_{\ell^{\prime}}}\left[-\frac{\partial G(\hat{\mathbf{r}}-\mathbf{r})}{\partial n(\mathbf{r})} \psi_{\text {ext }}(\mathbf{r})+G(\hat{\mathbf{r}}-\mathbf{r}) \frac{\partial \psi_{\text {ext }}}{\partial n(\mathbf{r})}(\mathbf{r})\right] d s(\mathbf{r}) \\
=\sum_{m=-\infty}^{\infty} e^{-\sqrt{-\lambda_{m}}\left(\hat{x}-x_{\ell}^{+}\right)+i(m+\beta) \hat{y}} L_{m, \ell}^{+}\left(\psi_{\text {ext }}, \partial_{n} \psi_{\text {ext }}\right) .
\end{gathered}
$$


If instead $\hat{x}<x$ for all $(x, y) \in \partial D_{\ell}$, then the integral in (2.5a), restricted to $\bigcup_{\ell^{\prime}>\ell} \partial D_{\ell^{\prime}}$, becomes

$$
\begin{gathered}
\sum_{\ell^{\prime}=\ell}^{L} \int_{\partial D_{\ell^{\prime}}}\left[-\frac{\partial G(\hat{\mathbf{r}}-\mathbf{r})}{\partial n(\mathbf{r})} \psi_{\text {ext }}(\mathbf{r})+G(\hat{\mathbf{r}}-\mathbf{r}) \partial_{n} \psi_{\text {ext }}(\mathbf{r})\right] d s(\mathbf{r}) \\
=\sum_{m=-\infty}^{\infty} e^{\sqrt{-\lambda_{m}}\left(\hat{x}-x_{\ell}^{-}\right)+i(m+\beta) \hat{y}} L_{m, \ell}^{-}\left(\psi_{\text {ext }}, \partial_{n} \psi_{\text {ext }}\right) .
\end{gathered}
$$

By using the reference points $\mathbf{r}_{\ell}^{ \pm}$, we compute exponentials only of quantities having a negative real part, both in the definition of $L_{m, \ell}^{ \pm}$and in the separated factors involving $\hat{\mathbf{r}}$.

Let us now augment the finite approximating system (3.1) by introducing the auxiliary variables

$$
\zeta_{m, \ell}^{ \pm}=L_{m, \ell}^{ \pm}\left(\sum a_{i} h_{i}, \sum b_{i} h_{i}\right)
$$

We then approximate $G$ using $M=2 m_{0}+1$ values of $m$ so that, for any sample point $\hat{\mathbf{r}}$ that is at a sufficient distance from group $\ell$ of rods, say $\hat{x}-x_{\ell}^{+}>\delta_{2}$, we may write

$$
\begin{gathered}
\sum_{i: \operatorname{supp}\left(h_{i}\right) \in \bigcup_{\ell^{\prime} \leq \ell} \partial D_{\ell^{\prime}}} \int\left[-\frac{\partial G(\hat{\mathbf{r}}-\mathbf{r})}{\partial n(\mathbf{r})} a_{i} h_{i}(\mathbf{r})+G(\mathbf{r}-\hat{\mathbf{r}}) b_{i} h_{i}(\mathbf{r})\right] d s(\mathbf{r}) \\
\approx \sum_{m=-m_{0}}^{m_{0}} e^{-\sqrt{-\lambda_{m}}\left|x_{\ell}^{ \pm}-\hat{x}\right|-i(m+\beta) \hat{y}} \zeta_{m, \ell}^{ \pm} .
\end{gathered}
$$

If $x_{\ell}^{-}-\hat{x}>\delta_{2}$, a similar approximation holds. In setting up the finite linear system, we make use of the following recursion relations for the variables $\zeta_{m, \ell}^{ \pm}$:

$$
\begin{aligned}
& \zeta_{m, \ell}^{+}=e^{-\sqrt{-\lambda_{m}}\left(x_{\ell}^{+}-x_{\ell-1}^{+}\right)} \zeta_{m, \ell-1}^{+}, \quad \ell=2, \ldots, L, \\
& \zeta_{m, \ell}^{-}=e^{-\sqrt{-\lambda_{m}}\left(x_{\ell+1}^{-}-x_{\ell}^{-}\right)} \zeta_{m, \ell+1}^{-}, \quad \ell=1, \ldots, L-1 .
\end{aligned}
$$

(Actually, $\zeta_{m, L}^{+}$and $\zeta_{m, 1}^{-}$are not needed.)

An example with $L=14$ is illustrated in Figure 3.2. The variables are arranged in the order $\left(a_{1}, b_{1}, \ldots, a_{N}, b_{N}, \zeta_{m, \ell}^{ \pm}\right)$, and the rows represent the left-hand sides of (3.1a), then of (3.1b), then the definitions (3.2) of the auxiliary variables $\zeta_{m, \ell}^{ \pm}$. The shaded areas represent nonzero entries.

The key features of this matrix are these:

- The upper left block-diagonal portion records interactions between sample points in one group of rods and elements in the same group through the external Green's function. When $L$ becomes large with the number of rods per group remaining small, this portion becomes increasingly sparse.

- In the lower portion defining the auxiliary variables, the number of nonzero entries scales only linearly with $L$.

- The upper right portion brings in the interactions between rods in different groups. The number of nonzero entries scales linearly in $L$.

- The middle block-diagonal structure becomes sparser as the number of rods increases; it represents interaction between sample points in one rod and elements in the same rod through the interior Green's functions. 

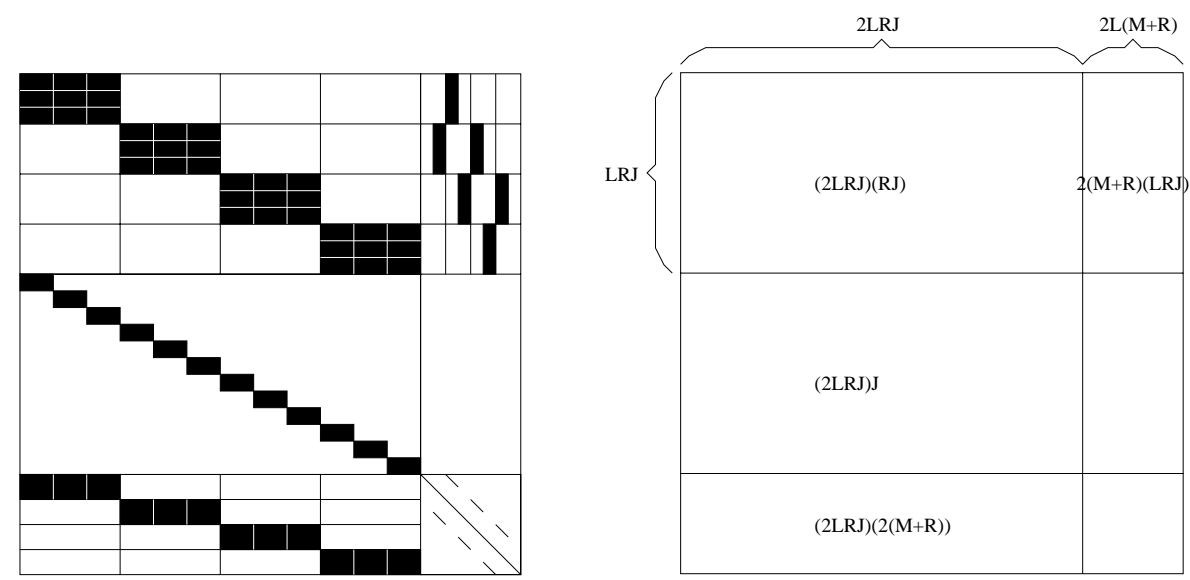

FIG. 3.2. The matrix for an $L \times R$ supercell of rods. $J$ is the number of basis elements and the number of sample points per rod, and $M$ is the number of Fourier modes used in the auxiliary variables $\zeta_{m, \ell}^{ \pm}$in the case for which $R=1$. The numbers in the figure on the right bound the number of nonzeros in each block of the matrix.

We have chosen, for the simulations in this paper, to solve the system using Gaussian elimination with partial pivoting. With this method, the auxiliary variables provide the most efficiency in the case in which the crystal consists of one horizontal row of rods repeated periodically in $y$.

Let us now consider crystals that consist of $L$ vertical groups of rods, each containing $R$ rods, each with $J$ nodes. Suppose that we wish to calculate transmission for actual incident frequencies up to a fixed maximal value. Since one period of the structure contains $R$ rods in the $y$-direction, the maximal value of the reduced frequency $k$ scales linearly in $R$, thereby increasing the number of penetrating (nondecaying) modes of the Fourier form of the exterior Green's function. The number of modes $M(R)$ used in the auxiliary variables must therefore also increase with $R$, say $M(R)=M+R$. Figure 3.2 illustrates this case, with the number of nonzero entries indicated as functions of $L, R$, and $J$.

If we fix $R$ and $J$ and use a Gaussian elimination method of solution, the multiplication count of our algorithm is a linear function of $L$. However, since the separability does not apply to the $y$-variable, it is not linear in $R$.

The separability can also be used to gain efficiency in the calculation of the matrix entries. Let $h_{i}$ be a basis function, and let $\mathbf{r}_{*}^{ \pm}=\left(x_{*}^{ \pm}, y_{*}\right)$ denote points on $\operatorname{supp}\left(h_{i}\right)$ such that $x_{*}^{+}=\max \left\{x:(x, y) \in \operatorname{supp}\left(h_{i}\right)\right\}$ and $x_{*}^{-}=\min \left\{x:(x, y) \in \operatorname{supp}\left(h_{i}\right)\right\}$. Then, for any sample point $\hat{\mathbf{r}}=(\hat{x}, \hat{y})$, one has

$$
\begin{aligned}
L_{1}\left(a_{i} h_{i}, b_{i} h_{i}\right)(\hat{\mathbf{r}})= & \sum_{m=-\infty}^{\infty} e^{-\sqrt{-\lambda_{m}}\left|\hat{x}-x_{*}^{ \pm}\right|+i(m+\beta)\left(\hat{y}-y_{*}^{ \pm}\right)} \\
& \times \frac{1}{4 \pi \sqrt{-\lambda_{m}}} \int_{\operatorname{supp}\left(h_{i}\right)} e^{-\sqrt{-\lambda_{m}}\left|x_{*}^{ \pm}-x\right|-i(m+\beta) y} \\
& \times\left[a_{i}+\left(\mp \sqrt{-\lambda_{m}} n_{x}(\mathbf{r})+i(m+\beta) n_{y}(\mathbf{r})\right) b_{i}\right] h(\mathbf{r}) d s(\mathbf{r}),
\end{aligned}
$$

in which $x_{*}^{+}\left(x_{*}^{-}\right)$is taken if $\hat{x}>x_{*}^{+}\left(\hat{x}<x_{*}^{-}\right)$. This means that, if the crystal contains several rods of the same shape and size and basis functions are chosen identically on 
all these rods, then the integrals in (3.4) need to be computed only once. They may then be used for each identical (but shifted) basis function and each sample point $\hat{\mathbf{r}}=(\hat{x}, \hat{y})$ such that $\delta_{1}<\hat{x}-x_{*}^{+}<\delta_{2}$ or $\delta_{1}<x_{*}^{-}-\hat{x}<\delta_{2}$. In addition, the computed integrals for all basis elements in a group of rods may be used to build the auxiliary variables $\zeta_{m, \ell}^{ \pm}$.

4. Computations and results. We use our computational model to simulate $E_{z}$ transmission (electric field normal to the $x y$-plane) through various crystals and to analyze the electric fields generated inside the crystals. Our primary concern is an investigation of the effects of introducing a periodic horizontal channel through the crystal. We study various types of resonances that arise, and consider a theoretical understanding of them. We also show some simulations of transmission through a perfect crystal and through a randomly perturbed crystal at all angles of incidence.

We compute the transmission coefficient in the following way: At a point $(\hat{x}, 0)$ located at a sufficient distance from the photonic crystal slab, we compute the penetrating plane-wave modes of the exterior field,

$$
\psi_{\mathrm{ext}}=\sum_{m=-\infty}^{\infty} c_{m} e^{-\sqrt{-\lambda_{m}} x+i(\beta+m) y},
$$

that is, the modes $m$ for which $\sqrt{-\lambda_{m}}$ is imaginary. The coefficients $c_{m}$ are given by

$$
c_{m} \approx \sum_{\ell=1}^{L} e^{-\sqrt{-\lambda_{m}}\left|x_{\ell}^{+}-\hat{x}\right|} \zeta_{m, \ell}^{+}+\delta_{\bar{m}, m} e^{-\sqrt{-\lambda_{m}} \hat{x}},
$$

in which $\delta$ is the Kronecker delta symbol. The square of the transmission coefficient is the ratio of the energy transmitted through the crystal, divided by the energy of the incident wave:

$$
T^{2}(k ; \theta)=\frac{\sum_{m=m_{1}}^{m_{2}}\left|c_{m}\right|^{2} \sqrt{\lambda_{m}}}{\sqrt{\lambda_{\bar{m}}}}
$$

where the sum is over propagating modes.

4.1. Advantages of the boundary-integral method. The boundary-integral method is particularly well suited to studying resonant phenomena. We have seen confirmation by a finite-difference time domain (FDTD) method [4] of all the features in the transmission graph in Figure 4.5; however, the sharper features could not be resolved in a reasonable amount of time. In addition, the shapes of the spikes that we observe in the transmission graph for a photonic crystal with a channel defect (see section 4.4) are not predictable, so the sharpness-enhancement methods used in FDTD schemes cannot be applied effectively.

Our method also provides us with a theoretical and numerical tool to study fields induced by arbitrary harmonic EM sources. In particular, it is interesting to investigate the existence of EM waves in the absence of any source at all. These are surface waves, which, in the case of photonic crystal slabs, would be EM states localized in the crystal and traveling along its length. We are now investigating these problems, which will be the subject of further communication.

4.2. Comparison of the first- and second-kind formulations. In our experiments, we have used both the first-kind system (2.5) and the second-kind system (2.6). In all the cases for which we have compared the two formulations, we find that 

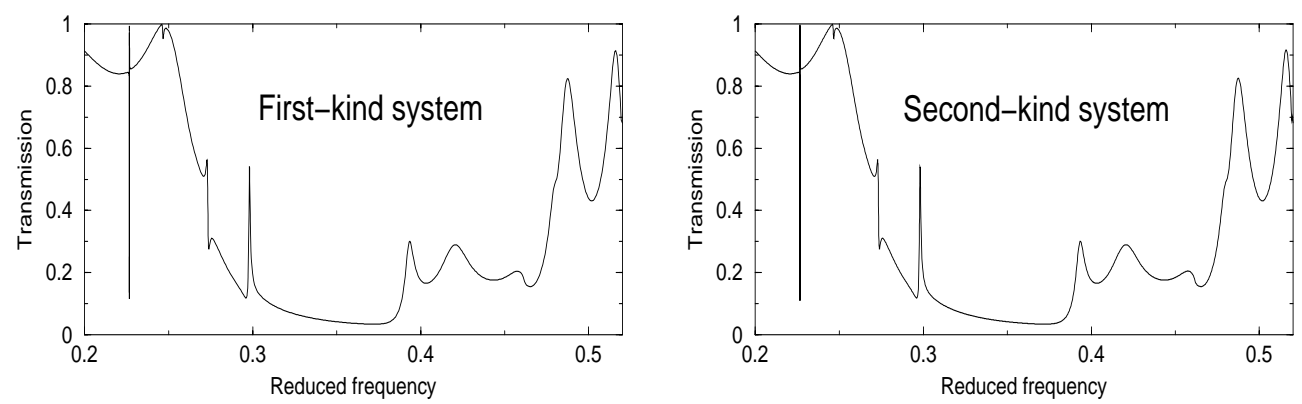

FIG. 4.1. A comparison between Fredholm of the first kind and Fredholm of the second kind results for the transmission graph of an E-polarized field through a $6 \times 4$ supercell with a channel defect of width 0.5 .

the transmission graphs are, practically speaking, identical. In particular, we observe that the spikes that appear in the transmission graph of a crystal with a channel defect are captured essentially identically with both systems. A comparison of results is shown in Figure 4.1.

The deviation between the results from the first-kind and second-kind systems is most apparent near the sharp features of the graph. Even so, with a discretization of 12 elements per rod, the two systems give a difference of less than $10^{-6}$ in the reduced frequency $k$ at which the spike (c) in Figure 4.5 occurs. The width of the spike at half its length is about $8 \times 10^{-6}$. The results for the transmission at the tip of the spike differ by about 0.005 , and the relative error between the results for the maximal amplitude of the field in the crystal is less than $0.15 \%$. Moreover, the two formulations produce contour plots of the fields that look identical.

Thus, we demonstrate that, for systems of the size that we consider in this paper, in which the matrix for the discretized system is typically around $1000 \times 1000$, the two formulations give practically identical results. In computing the quadrature integrals to set up the discrete system, it is faster to use the first-kind system. However, at very sharp resonances ((c) and (d) in Figure 4.5), we find that, to match the results obtained from the second-kind formulation at a certain discretization, a finer discretization of the first kind is required. Some of the figures shown in this section were computed using the first kind and others using the second; that information is indicated in the captions.

4.3. Bandgaps. We compute $E_{z}$ transmission through a perfect square lattice five rods thick at all angles of incidence for reduced frequencies in the range $(0,1)$ (Figure $4.2(\mathrm{a}))$. At normal incidence $(\theta=0)$, there are two frequency intervals for which transmission is practically zero. The lower of these intervals contains a "complete photonic bandgap," that is, an interval of electromagnetic frequencies at which the infinite crystal admits no propagating fields in any direction. This can be confirmed by comparison with the atlas of complete bandgaps in Joannopoulos, Meade, and Winn [5]. In Figure 4.2(a), one observes the persistence of the near-zero transmission for all angles of incidence in this frequency interval. The upper interval, on the other hand, is not a complete photonic bandgap, as varying the angle of incidence allows fields at these frequencies to be transmitted.

We then take a $5 \times 5$ supercell within the perfect crystal and randomly perturb the centers of the rods in both the $x$ - and $y$-variables. Figure 4.2 shows the transmission 

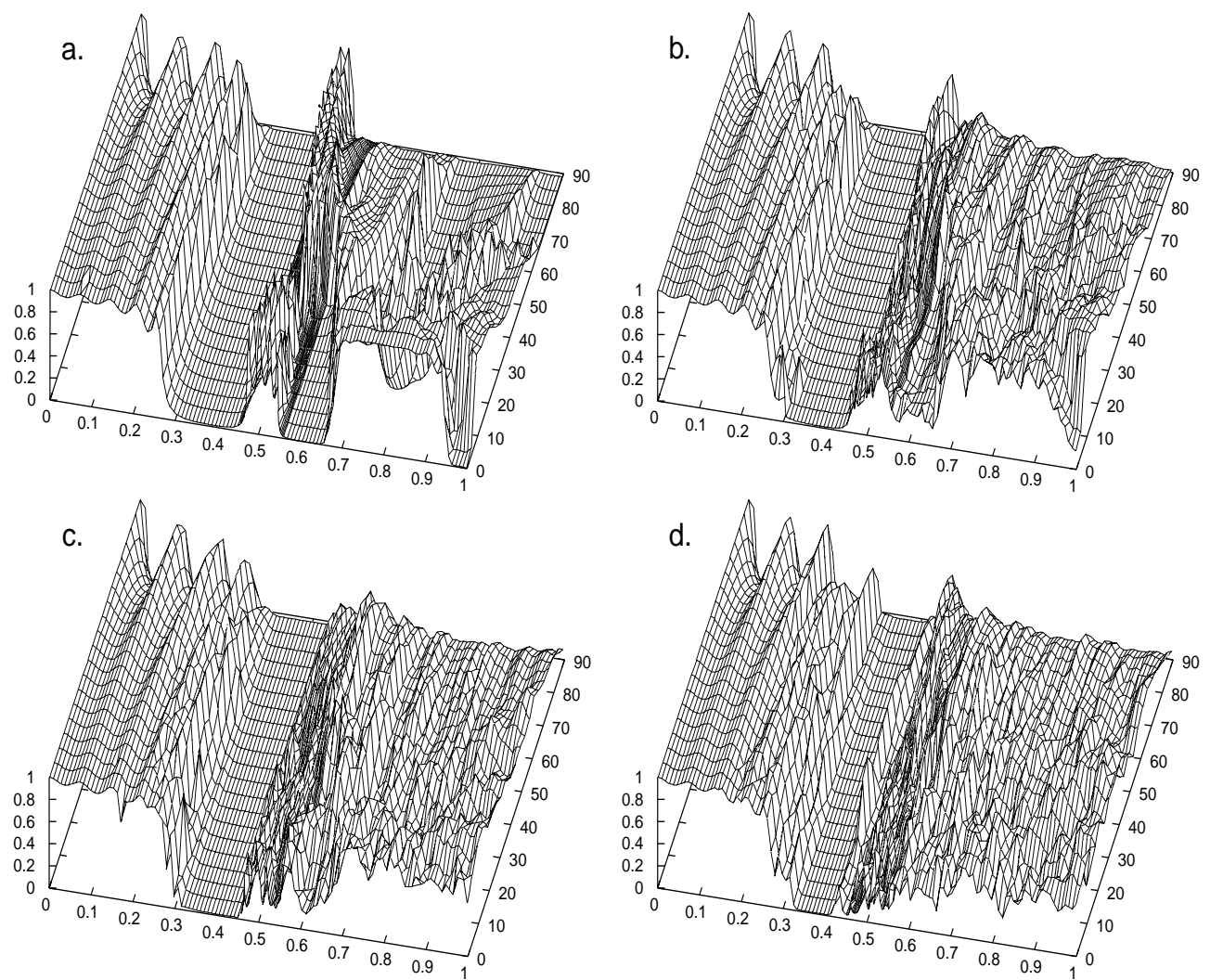

FIG. 4.2. Transmission through a square lattice with a randomly perturbed $5 \times 5$ supercell. The graphs show transmission as a function of frequency and angle of incidence: (a) no perturbation, (b) perturbation of the centers up to $10 \%$ of the lattice constant, (c) perturbation up to $20 \%$, (d) perturbation up to $30 \%$. The system of the first kind was used.

graph for all angles of incidence through three realizations, using different amounts of perturbation.

Our most striking observation is the robustness of the complete bandgap under perturbations. Whereas the incomplete gap disappears quickly under small random perturbations, the complete gap persists even for perturbations up to $30 \%$ of the lattice constant. Although the frequency interval over which the transmission is practically zero shrinks with increasing perturbation, all of our realizations have shown the persistence of an interval for which transmission is blocked for all angles of incidence.

In [6], various types of perturbations are considered, and the bandgap interval for both $E_{z}$ and $H_{z}$ waves is studied as the amount of perturbation increases.

4.4. Lattice with a channel defect. We analyze $E_{z}$ EM scattering in a $y$ periodic structure with a periodic channel defect, as depicted in Figure 4.3. In contrast to the Fabry-Perot cavity considered in [8], the cavity in the current structure is parallel to the $x$-axis, resulting in more complex $2 \mathrm{D}$ interactions of EM waves in the structure. Our primary interest in this study is the characterization of resonant frequencies that arise in the transmission coefficient. We limit our analysis to the case of normal incidence.

We compute the transmission coefficient $T(k)$ using several values of the channel 


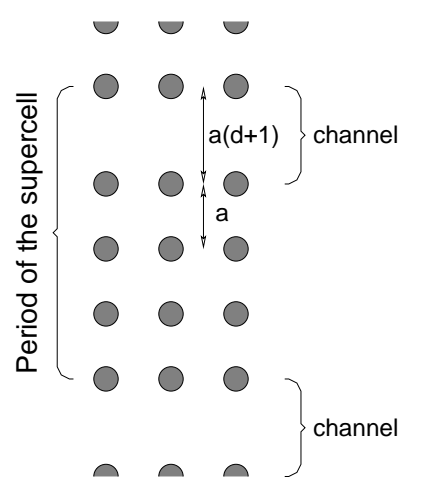

FIG. 4.3. A crystal with a channel defect of size $d$ in $a 4 \times 3$ supercell. In the numerical calculations, the period of the supercell is scaled to $2 \pi$.
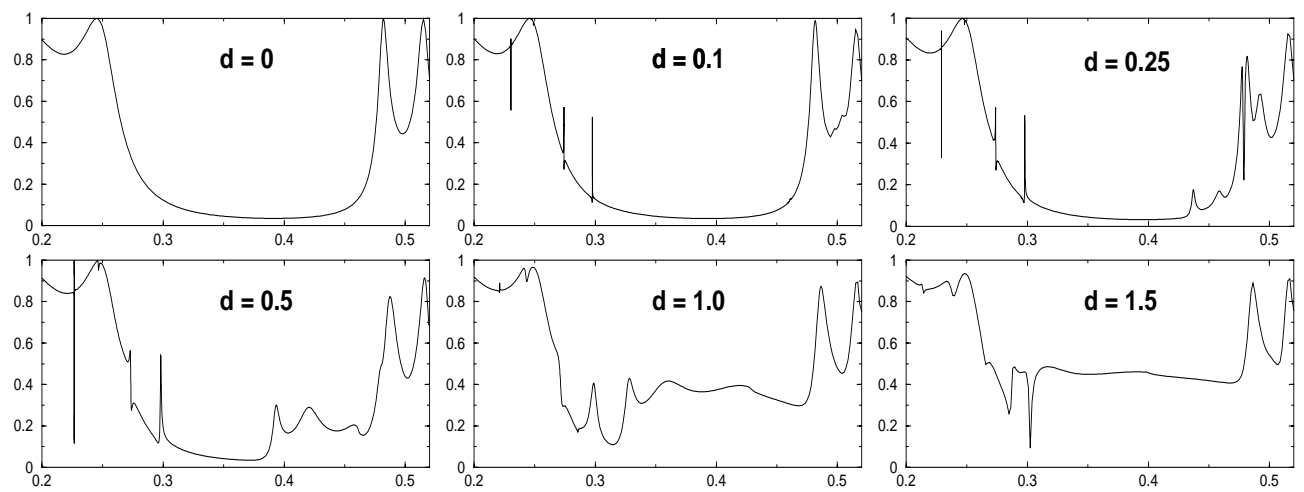

FIG. 4.4. Transmission of $E_{z}$ fields of normal incidence through crystals with channel defects of various sizes $d$ in a $6 \times 4$ supercell. Relative transmission $T$ is plotted against the reduced frequency $k$ with $\theta=0$. The system of the first kind was used.

width $d$ in a $6 \times 4$ supercell of circular dielectric rods (Figure 4.4). The ratio of the radius of the rods to the distance between closest rods is $1 / 2 \pi$. We use eight elements (sixteen nodes) per rod in the discretization, with eight quadrature points per element in the integrations.

In plotting the transmission, we make physically meaningful comparisons of scattering in the channel structure to scattering in the defect-free structure by fixing our definition of the reduced frequency to be $k=\omega a /(2 \pi c)$, where $a$ is the lattice constant or the distance between nearest rod centers. The introduction of a periodic channel into the otherwise perfect structure results in "resonances" that are manifest in the transmission graph as various sorts of spikes and humps that are absent in the transmission graph of the perfect crystal. At these special frequencies, the electric field in the crystal assumes various characteristics that are not present at a typical frequency. The most notable of these are amplification and a departure from the typical field pattern that resembles generally $x$-directional wave interference patterns. (Figure 4.5(a) shows a typical field pattern in a near-transmission region of the lattice with a channel.) We find three categories of resonances in the channel structure. All of these categories have been confirmed by a finite-difference time domain numerical 

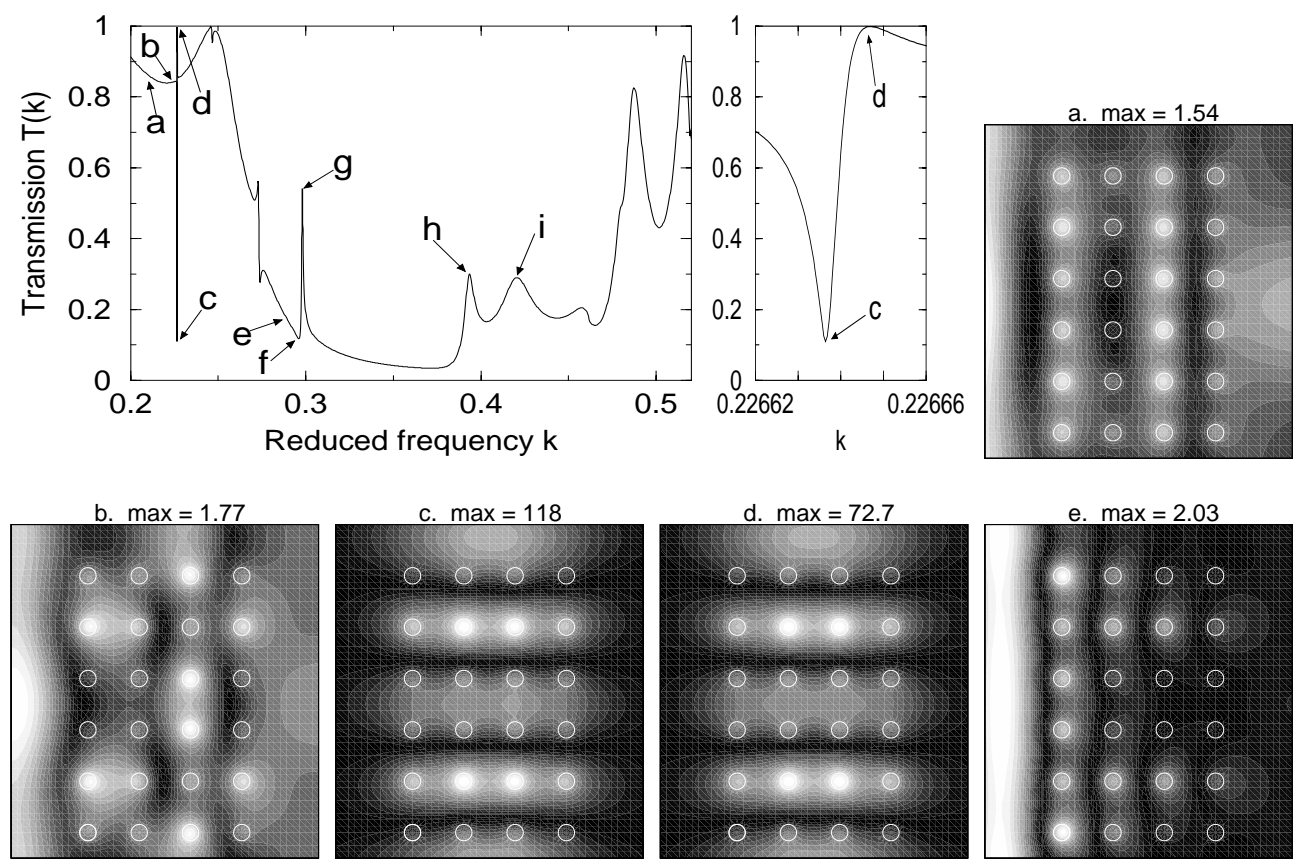

e. $\max =2.03$
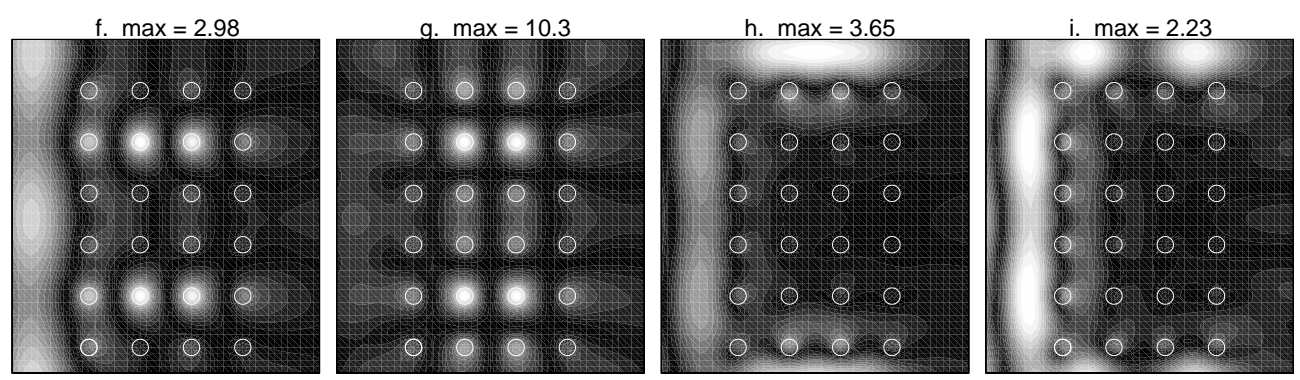

FIG. 4.5. The electric field produced by $E_{z}$ fields normally incident to a crystal with a channel defect of size $d=0.5$ in a $6 \times 4$ supercell. One period of the crystal is shown, with the channel at the top. The grey-scale indicates the magnitude of the electric field, with white representing the highest value, and its maximum amplitude relative to the incident field is shown above each figure. The system of the second kind was used.

method [4].

The first category of resonances (Figure $4.5(\mathrm{~h}, \mathrm{i})$ ) appear at higher frequencies of the bandgap and are characterized by local maxima in the transmission graph. They are those that one might expect in a channel structure. Plots of the electric fields in the structure at these frequencies typically show a low amplitude throughout the crystal except for the area in the channel, in which larger-amplitude resonating fields are present. We refer to these resonances as waveguide modes. The transmission maxima at these resonances are diffuse and shift to lower frequencies as the channel width $d$ increases (Figure 4.4). With increasing $d$, the transmission also increases as electromagnetic waves propagate more freely through the channel with the resonances eventually disappearing. The low quality factor ( $Q$-value) of these resonances indicates that they are of limited practical interest in the design of photonic crystal filters. (The $Q$-value is defined as $Q=k / \Delta k$, where $\Delta k$ is the width of the spike at 
half of its height.)

The other two categories of resonances appear at the left end of the bandgap (second category, Figure 4.5(f, g)) and to the left of the gap in the first band (third category, Figure 4.5(c,d)). These are typically dual spikes (a sharp dip followed by a sharp peak, or the reverse) that, in contrast to the waveguide modes, exhibit higher quality factors and attain their peak amplitude inside certain of the rods.

Resonances of the second category are characterized chiefly by an upward spike and a smaller downward spike in the transmission graph and by a greatly amplified electric field that has a genuinely $2 \mathrm{D}$ structure. We have observed more than a tenfold amplification of the electric field.

The third category of resonances is characterized by a very sharp dual spike within the near-transmission band. It is remarkable that the electric fields at these spikes exhibit peak amplitudes as large as 170 times the incident amplitude and resemble an interference pattern of waves propagating normal to the incident wave (in the $y$ direction). A typical field with frequency in the band generally resembles interference patterns of waves propagating in the $x$-direction.

In the most extreme cases ((c) and (d) of Figure 4.5), numerical computations of the fields in the crystal produce essentially standing fields with oscillations running in the $y$-direction. We make this more precise: The physically meaningful electric field $E(x, y)=\operatorname{Re}\left(\psi(x, y) e^{-i \omega t}\right)$ is the steady-state field induced by the incident source $\cos (k x-\omega t)$. In the case of the upward spike (d), we find that $\operatorname{Im}(\psi(x, y))$ is nearly zero, so that $\psi(x, y)$ is practically a real function, and $E(x, y) \approx \psi(x, y) \cos (\omega t)$ a standing field. Moreover, $\psi(x, y)$ is equal to zero on nearly horizontal lines, and the peaks and troughs of the field appear as horizontal light bands in Figure 4.5(d). In the case of the downward spike (c), the situation is the same, except that now $\operatorname{Re}(\psi(x, y))$ is practically zero and $E(x, y) \approx-i \psi(x, y) \sin (\omega t)$. Thus there is a phase angle difference of $\pi / 2$ between the fields at these two spikes.

These resonances are of particular interest, as they exhibit very high quality factors and may provide a useful filtering property in that the structure can reflect incident radiation at a particular frequency while allowing neighboring frequencies to penetrate the lattice. In the most extreme case that we have observed $(d=0.5)$, we see an extremely narrow frequency interval with a minimal transmission of about $11 \%$, whereas neighboring frequencies are about $85 \%$ transmitted. At the frequency of maximal reflection, the field is amplified over a hundredfold inside the crystal, and we estimate the quality factor at around $2 \times 10^{5}$. The design of photonic crystal structures with such a filtering property has been considered in work on channel drop filters, in which two waveguides are separated by a photonic crystal resonator consisting of a periodic lattice with a cavity defect [2], [3].

4.5. Theoretical considerations. The electric fields at resonances of the third category resemble fields that are localized, or bound states, in the $x$-variable. A bound state of the Helmholtz equation exists in the absence of a source and thus must occur at a frequency for which the integral system has a nullspace. This is a frequency within the continuous spectrum of the Helmholtz equation for which a bound state exists in addition to the usual extended states. One may theorize that resonances of the third category occur at such frequencies.

A simplified problem in which the analysis is more tractable can give us insight into this eigenvalue problem. In fact, for the related problem in which we replace our photonic crystal slab by a solid slab of uniform dielectric material that is infinite in the $y$-direction and finite in the $x$-direction, we can prove the existence of solutions of 


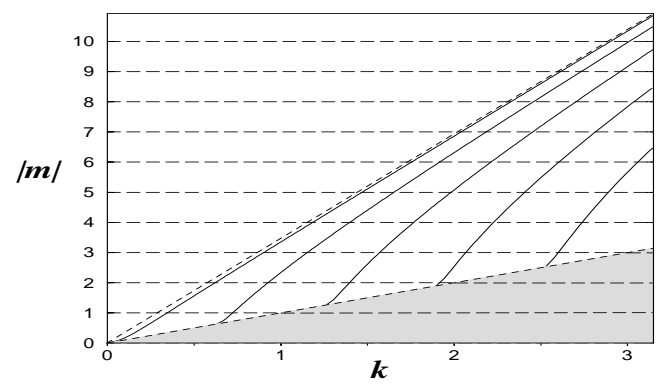

FIG. 4.6. The spectrum of the Helmholtz equation for a slab of thickness 3 of uniform dielectric permittivity 12. The grey area covers $(k, m)$ pairs for which there is an extended solution of the form $\phi(x) e^{i m y}$. The curves trace out $(k, m)$ pairs for which there exist additional solutions of the same form that decay as $|x| \rightarrow \infty$. The lower dotted line is $|m|=k$, and the upper one is $|m|=\sqrt{12} k$.

the Helmholtz equation that are localized in the dielectric. The Helmholtz equation becomes

$$
\left(\partial_{x}^{2}+\partial_{y}^{2}\right) \psi+k^{2} \epsilon(x) \psi=0,
$$

in which $\epsilon(x)=\epsilon_{0}$ within the slab and $\epsilon(x)=1$ without. If we seek solutions of the separable form

$$
\psi(x, y)=\phi(x) e^{i m y},
$$

we arrive at an eigenvalue problem for the linear Schrödinger operator applied to $\phi$ :

$$
\phi^{\prime \prime}(x)+k^{2} \epsilon(x) \phi(x)=m^{2} \phi(x) .
$$

We see that extended electromagnetic states exist for values of $m$ such that $m^{2}<$ $k^{2}$ (the continuous spectrum). In the interval $k^{2} \leq m^{2}<\epsilon_{0} k^{2}$, there exist a finite number of values of $m$ for which there is a bound state $\phi(x)$, that is, a solution that decays exponentially as $|x| \rightarrow \infty$ (the point spectrum) (Figure 4.6).

Although the idea of a supercell is not naturally defined for the uniform slab, we may artificially impose a period of $2 \pi$ in the $y$-direction to relate to the slab of photonic crystal. Thus we consider solutions of the form $\phi(x) e^{i m y}$ for integral values of $m$. The point-spectrum values of $m$ vary continuously with $k$ and necessarily, for certain values of $k$, attain integer values. At these values of $k$, we have extended $y$ periodic solutions of the Helmholtz equation for $|m|<k$ and additional bound states $\phi(x) e^{ \pm i m y}$ for some integer $m$ such that $k<|m|<\epsilon_{0} k$. For example, in Figure 4.6, at $k \approx 1.75$, there exist solutions $\phi_{m}(x) e^{i m y}$ that are extended electric fields for $m=-1,0,1$ and bound fields for $m=-4,4$.

Based on these observations for the uniform slab, we conjecture that such bound states exist for slabs of a photonic crystal material. In our channel experiments, we find that the condition number of the matrix for the discretized system increases at the resonant frequencies and that the amount of increase depends roughly on the sharpness of the anomaly in the transmission graph. At the resonance (c) in Figure 4.5, with eight elements per rod, the condition number goes up to around $10^{6}$ compared to neighboring frequencies at which the condition number is around $10^{3}$. The matrix at the resonance has a small eigenvalue (of magnitude $\approx 10^{-5}$ ), but we cannot resolve numerically whether the system of integral equations has a zero eigenvalue. 

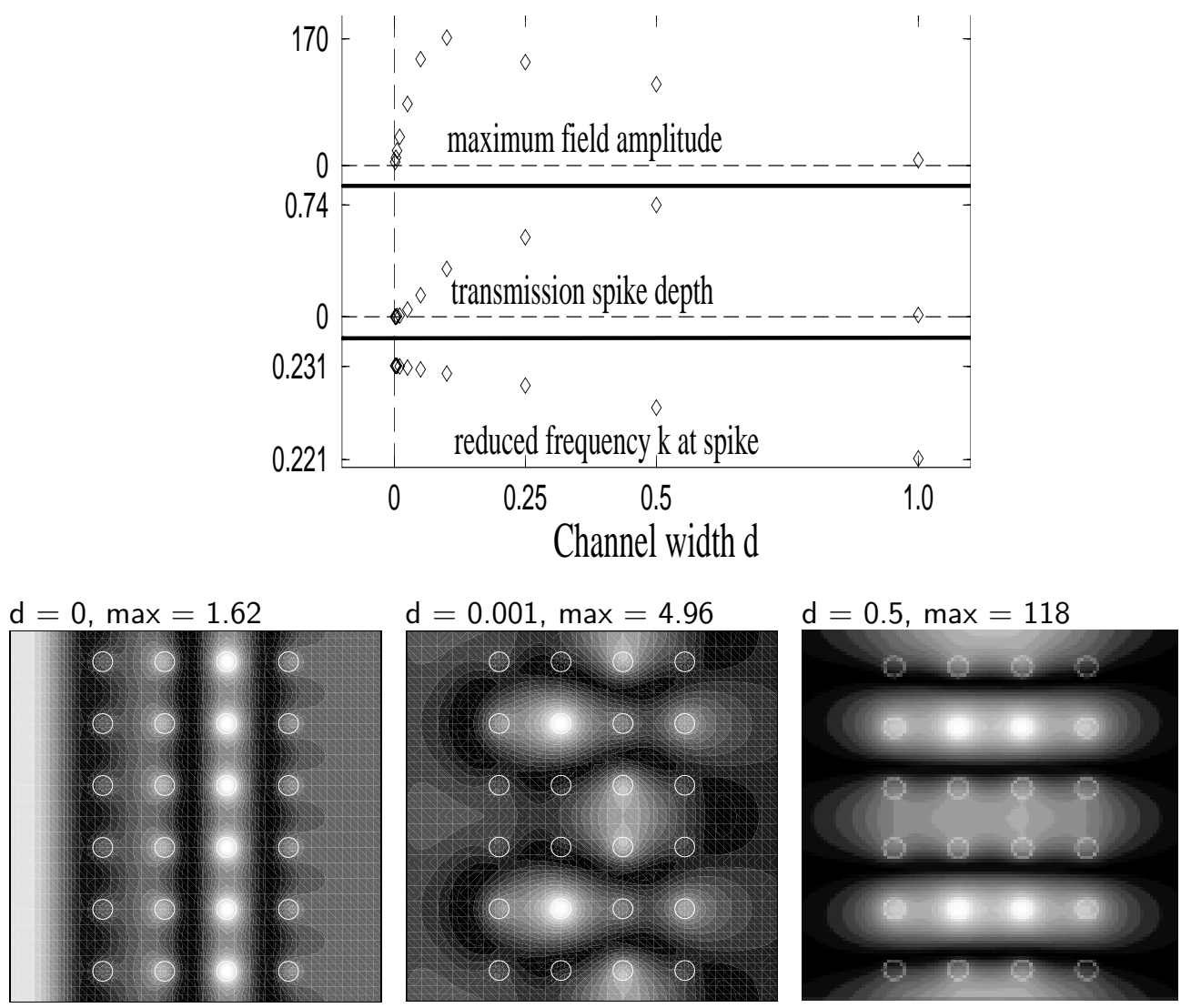

FIG. 4.7. These data track a third-category spike for various values of the channel width d. For $d=0.5$, it is resonance $c$ in Figure 4.5. The three field simulations show how the direction of the field changes as the channel is introduced. Even a tiny defect of $d=0.001$ produces a significant change in the field. The maximum amplitude of the field is indicated above each figure. The system of the first kind was used.

As the channel width decreases, the resonant frequency approaches a limiting value, while the depth of the spike diminishes to zero. At the same time, the phenomena of field amplification and the resemblance to $y$-directional interference patterns disappear, and the fields resume their typical $x$-directional appearance with no considerable amplification (Figure 4.7). Thus we demonstrate that these resonances are a consequence of the channel. Whether they can exist only in the presence of an electromagnetic source (i.e., the integral system has a nontrivial nullspace), however, is unclear.

We have done similar simulations of transmission through crystal slabs that have periodic defects. We have studied channel defects in a $6 \times 3$ and $6 \times 5$ supercell and other defects such as the removal of just one rod from the supercell. Similar resonances appear, and their locations differ from structure to structure. Thus it seems that at least the second and third categories of resonances are a consequence of a general periodic defect.

We see a similar resonance in another numerical experiment, in which we simulate scattering through a defect-free crystal that is one rod thick with an unphysically high dielectric contrast (Figure 4.8). We observe an increase in the condition number of 

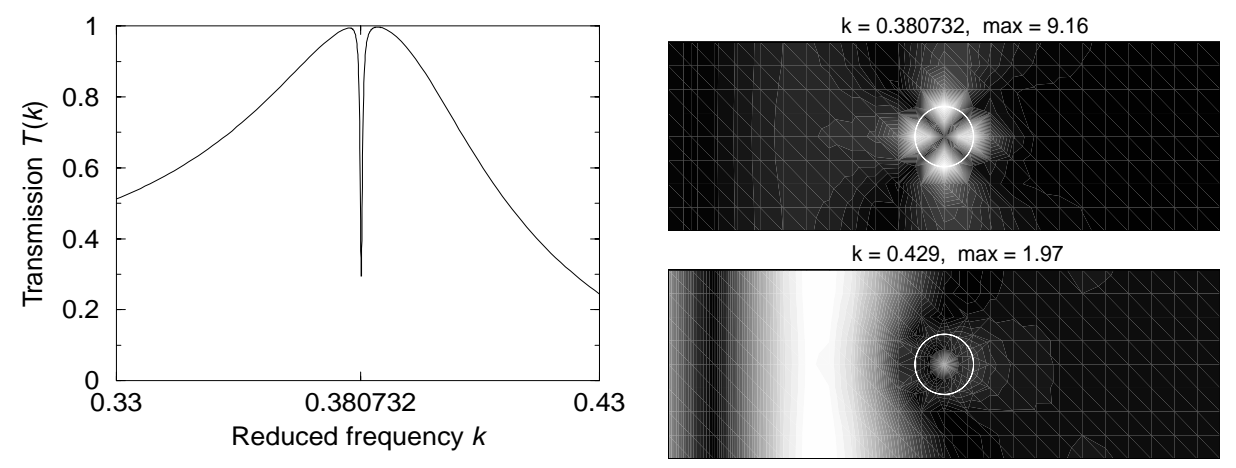

FIG. 4.8. $E_{z}$ transmission through a crystal that is one rod thick with dielectric contrast 100. The field produced by an incident wave at the resonant frequency is shown (top right) along with the field for a typical frequency of low transmission (bottom right). The maximum amplitude of the field is indicated. The system of the second kind was used.

the discretized integral system at the frequency that corresponds to a downward spike in the transmission graph and a high-amplitude localized field inside the rods. Again, it is not clear that the integral system has a nontrivial nullspace at this resonant frequency.

We are now making a theoretical and numerical study of bound states and resonances, which will be the subject of further communication.

Acknowledgment. This study benefitted from several fruitful discussions with V. Papanicolaou.

\section{REFERENCES}

[1] M. M. Beaky, J. B. Burk, H. O. Everitt, M. A. Haider, and S. Venakides, Two dimensional photonic crystal Fabry-Perot resonators with lossy dielectrics, IEEE Trans. Microwave Theory Techniques, 47 (1999), pp. 2085-2091.

[2] S. Fan, P. R. Villeneuve, J. D. Joannopoulos, and H. A. Haus, Channel drop filters in photonic crystals, Optics Express, 3 (1998), pp. 4-11.

[3] S. Fan, P. R. Villeneuve, J. D. Joannopoulos, and H. A. Haus, Channel drop tunneling through localized states, Phys. Rev. Lett., 80 (1998), pp. 960-963.

[4] C. Hale, personal communication, Mathematics Department, Duke University, Durham, NC, 2000.

[5] J. D. Joannopoulos, R. D. Meade, and J. N. Winn, Photonic Crystals: Molding the Flow of Light, Princeton University Press, Princeton, NJ, 1995.

[6] E. Lidorikis, M. M. Sigalas, E. N. Economou, and C. M. Soukoulis, Gap deformation and classical wave localization in disordered two-dimensional photonic-band-gap materials, Phys. Rev. B, 61 (2000), pp. 13458-13464.

[7] C. MüLLER, Foundations of the Mathematical Theory of Electromagnetic Waves, SpringerVerlag, Berlin, New York, 1969.

[8] S. Venakides, M. A. Haider, and V. Papanicolaou, Boundary integral calculations of twodimensional electromagnetic scattering by photonic crystal Fabry-Perot structures, SIAM J. Appl. Math, 60 (2000), pp. 1686-1706.

[9] E. Yablonovitch, Photonic band-gap structures, J. Opt. Soc. Amer. B Opt. Phys., 10 (1993), pp. 283-295. 